\title{
Histoire de la genèse du procédé de fibrage du verre
} TEL

\author{
History of the TEL glass fiber method
}

\author{
Marianne Chouteau, Joelle Forest, Céline Nguyen ${ }^{1}$ \\ ${ }^{1}$ EA 4148 - S2HEP, Université de Lyon, France, marianne.chouteau@insa-lyon.fr; joelle.forest@insa-lyon.fr; \\ celine.nguyen@insa-lyon.fr
}

RÉSUMÉ. Cet article relate la genèse d'une innovation d'ISOVER (marque de la compagnie Saint Gobain) baptisée le procédé TEL, procédé de fibrage de la fibre de verre né dans les années 1950 en France. La biographie de ce procédé nous invite à suivre les traces d'une formidable épopée et aventure humaines. Elle révèle que cette innovation résulte d'un processus sinueux au cours duquel des hommes hors normes vont jouer un rôle clé. Chemin faisant, cette biographie nous conduit à nous émanciper du mythe de l'innovateur solitaire et d'une conception magique et spontanée de l'innovation. De même, elle conduit à prendre acte du fait qu'une innovation ne nait pas dans un désert et à considérer son épaisseur sociale. De la sorte, la biographie du TEL contribue au développement de connaissances sur le mode d'existence des innovations, troisième niveau de la culture technique. En cela, nous voyons avec l'exemple du TEL comment la biographie d'innovation peut contribuer au développement d'une culture technique.

ABSTRACT. This paper presentsthe history of the TEL method, an innovation developed in the 1950s by ISOVER - a brand of the French company Saint Gobain - to produce glass fiber. The process's history invites us to follow in the footsteps of a formidable human epic and adventureAt the same time, the TEL story proves that the lone innovator is a myth and that the innovation process is not magical and spontaneous. Similarly, it leads us to recognize the fact that innovation does not come out of nowhere, meaning that we need to consider its social depth. In this way, the history of the TEL method enables us to develop knowledge of how innovations exist, the third level of what we call "technical culture". We therefore see, with the TEL example, how the story of innovation can contribute to the development of a technical culture.

MOTS-CLÉS. Biographie, culture technique, processus créatif, processus de conception, Saint Gobain.

KEYWORDS. History, creative process, innovation process, technical culture, Saint Gobain.

\section{Introduction}

La question du froid a toujours été centrale pour la survie de l'être humain. S'en protéger par divers moyens, comme les vêtements ou les abris ont constitué autant de solutions de principe expérimentées par l'espèce humaine. Lorsqu'il s'est agi de bâtiment, l'épaisseur des matériaux de construction a d'abord été pensée comme la solution la plus performante, avant qu'on ne découvre le pouvoir isolant des matériaux creux, à l'instar de la brique, et qu'on ne crée des matériaux isolants dédiés. Entrent dans cette catégorie les fibres d'isolation, dont la laine de verre. Concrètement, il s'agit d'un enchevêtrement désordonné de fibres de verre très fines qui forme un feutre/matelas et qui emprisonne l'air (ce dernier représente plus de 95\% du volume du produit). Du fait de cette structure, la fibre de verre devient un excellent isolant thermique. Elle présente en outre l'intérêt d'être imputrescible et incombustible. C'est enfin un matériau qui n'est pas attaqué par les rongeurs et qui est relativement stable dans le temps.

Nos recherches conduites dans le cadre de la chaire Saint Gobain-INSA Lyon Ingénieurs ingénieux, se sont attachées à comprendre la genèse d'un procédé de fabrication de la laine de verre particulier, le procédé $\mathrm{TEL}^{1}$ à partir du processus qui l'a vu naître à savoir son processus de

\footnotetext{
${ }^{1}$ Le nom de ce procédé provient du fait qu'au cours de son invention, les ingénieurs impliqués dans le processus de conception ont retourné l'assiette d'un autre procédé nommé jusqu'alors LET. En inversant le fonctionnement du procédé, les ingénieurs ont 
conception. Nous nous inscrivons en effet dans la perspective artificialiste qui postule que connaître un artefact, donc une innovation, c'est connaître la façon dont il a été conçu. Ce qui, en d'autres termes, revient à souligner que la cible de la connaissance n'est pas « l'objet» (l'innovation dans sa réalité existentielle) en tant que tel, mais le " processus » qui l'a vu naître. L'accent mis sur le processus de conception conduit à considérer la "vie" de l'innovation (comment elle naît, se développe, l'environnement dans lequel il se déploie, les acteurs en présence, les grandes étapes de sa «vie », etc.) et à effectuer, ce faisant, non une biographie d'innovateur, comme cela se fait habituellement mais une biographie d'innovation [FOR 17a].

Pour ce faire, nous avons constitué un corpus d'une soixantaine de documents issus des archives de Saint-Gobain situées à Blois (France). A partir d'une liste des documents relatifs au TEL fournie par le service des archives de Saint-Gobain, nous avons sélectionné les cotes d'archives nous semblant les plus pertinentes pour comprendre à la fois le procédé mais aussi sa genèse. Sur place, nous avons opéré une seconde sélection en retenant et en photographiant les documents à première vue les plus pertinents. Ils ont des statuts relativement différents. Il s'agit tantôt de cahiers de laboratoire rédigés au cours du processus, d'interviews d'anciens salariés, de documents commémoratifs lors d'anniversaire (50 ans du TEL par exemple) de compte-rendus de groupement, etc. Ces documents ont été produits sur une période relativement longue, qui va des années 1920 pour les premiers brevets jusqu'à 2007. A ce matériau s'ajoutent quelques documents que la direction générale nous a transmis suite à notre demande et un entretien le 25 mai 2018 avec Jean Battigelli, acteur du développement du TEL, réalisé en juin 2018 par nos soins.

L'étude de ce corpus nous a permis de retracer la biographie du procédé TEL, et ce faisant d'extraire un certain nombre d'enseignements sur le mode d'existence d'une innovation. La première partie permet de voir les liens qui unissent l'innovation au contexte qui préside à sa genèse. Précisément, si cette biographie confirme qu'une innovation répond à un besoin, elle souligne aussi que celle-ci n'émerge pas dans un "désert technologique" et est affaire de stratégie d'entreprise. La seconde partie montre quant à elle que la genèse du TEL fut loin d'être un long fleuve tranquille et matérialise des décisions prises par des acteurs clés. Chemin faisant, nous verrons que la biographie d'innovation contribue au développement de la culture technique pensée aux trois niveaux que sont celui des connaissances relatives à la structure de l'objet, aux relations entre les différents modules qui le composent, celui des connaissances et savoir-faire développés et mobilisés pour user des objets techniques qui nous entourent, celui enfin ensemble des savoirs sur les modes d'existence des objets techniques qui permettent de penser et concevoir lesdits objets et le sens dont ils sont porteurs [CHO 17] $]^{2}$.

\section{Eléments de contexte}

\subsection{Une réponse à de nouveaux besoins et une préoccupation pour les usagers}

La seconde moitié du XIXe siècle est marquée par l'essor d'une marine marchande française qui s'internationalise. Plusieurs facteurs expliquent cet essor, dont le développement de la propulsion à vapeur qui permet de gagner en vitesse et en régularité, ainsi que l'intensification des échanges commerciaux de marchandises. On ne saurait oublier l'impact de la concession, à partir de 1851, des services maritimes postaux. Comme l'a souligné Berneron-Couvenhes [BER 07], elle a permis à la Compagnie des Messageries nationales de passer d'une flotte de 15 navires en 1851 à plus de 60 en 1870. Or, avec l'essor de la marine marchande, le besoin d'isolants thermiques légers se fait ressentir. Elle a en effet besoin d'un matériau souple, léger et résistant aux vibrations des machines

\footnotetext{
${ }^{2}$ La culture technique ainsi conçue conduit à s'émanciper d'une conception restrictive de la culture technique réduite au seul premier niveau, ou pour le dire autrement se démarquer d'une culture technique réduite à la seule approche fonctionnelle qui conduit à occulter l'épaisseur sociale de la technique et constitue un terreau favorable à des controverses stériles entre technophobes et technophiles qui à leur tour sont le terreau favorable à la diffusion de discours idéologiques.
} 
des navires [DAR 07]. La réponse tarde. L'industrie de la fibre de verre ne fait qu'une timide apparition en France au début des années 1930. Son développement demeure relativement faible jusqu'en 1945 (4 à 5 tonnes par jour), notamment parce que les "chantiers navals alors en léthargie constituaient encore à l'époque l'essentiel de notre clientèle" [BUL 67]. L'industrie de la fibre ne dépasse ainsi pas le stade semi industriel.

En France, l'après-guerre marque le début d'une période favorable aux matériaux d'isolation. Le pays est dévasté. Il faut rebâtir les usines et les bâtiments détruits. Ces deux domaines qui assurent des débouchés importants pour un isolant de bonne qualité thermique. Cet essor se poursuivra pour atteindre une augmentation de 30\% par an dans les années 1950 [COM 57]. L'entreprise SaintGobain enregistre un bénéfice record en 1951 de 1385 millions contre 585 millions de francs en 1950. Le développement de la construction a stimulé l'activité ; les ventes de fibres de verre ont augmenté de 50\% [CHO 60, p. 93]. La demande explose : "Exode rural, urbanisation et croissance économique se conjuguent pour faire exploser la construction de logements" peut-on lire dans Saint-Gobain et la laine de verre [ISO 2007, p. 76] à propos de l'histoire de la laine de verre. Cette explosion tient également au "développement de l'idée de l'isolation" [EXP 57, p. 1-2] qui transparaît dans les normes de la "construction de logements" et qui répond à un "souci d'économie de calories" [EXP 57, p. 2]. Cette économie de calories est le résultat d'une "augmentation constante du prix des fluides" [EXP 57, p. 2] dans un contexte ou la qualité du toucher de la laine de verre s'est grandement améliorée.

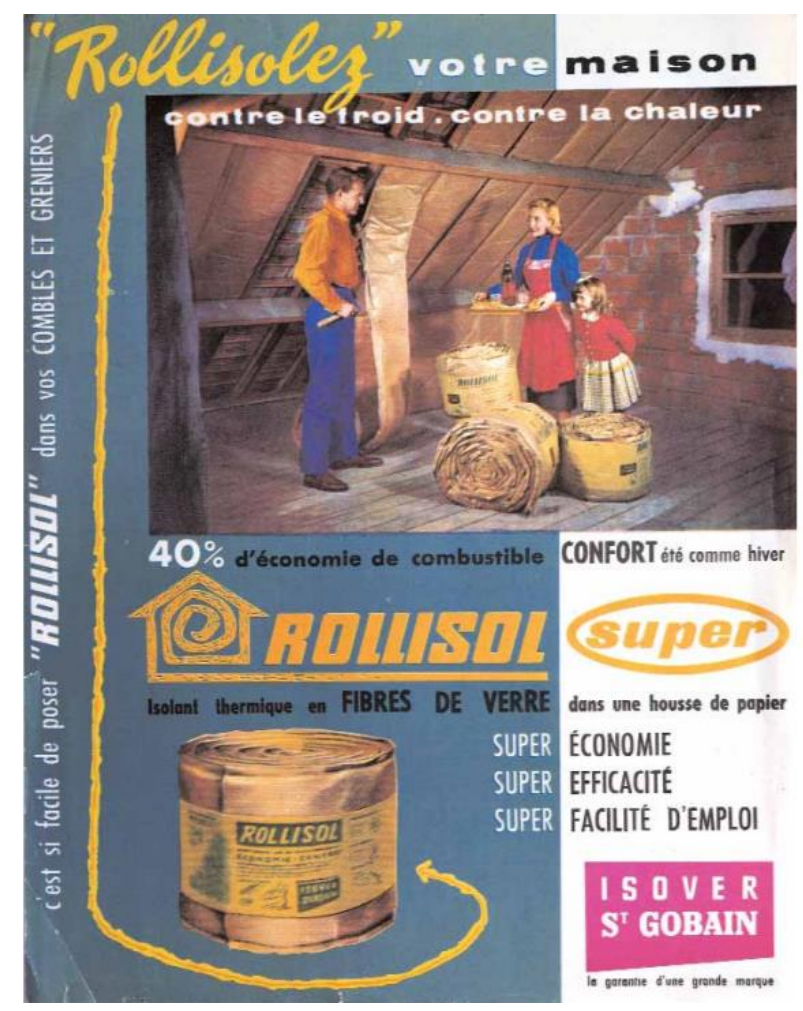

Illustration 1. Publicité Saint Gobain [ISO 07, p. 77]

En France, le besoin en isolant s'explique aussi par l'effet des campagnes publicitaires menées dans les années 1950, notamment la "campagne du silence" lancée pour développer le marché lié à l'acoustique [EXP 57, p. 2]. Progressivement, la laine de verre devient la technique préférée en France:

«L'isolation n'est plus une mode mais une nécessité atteignant toutes les formes d'activité. Pour répondre à cette nécessité la fibre de verre a été portée par la confiance des utilisateurs à la première place de tous les isolants en France. [...] le succès incontesté de ce matériau est justifié par ses résultats obtenus dans l'isolation thermique et phonique des bâtiments » (Etudes statistiques, 1957, p. 1). 
peut-on lire dans le rapport "Principes de fabrication de la fibre de verre" du 12 septembre 1957 rédigé vraisemblablement au sein de l'usine de Rantigny [ETU 57]. En effet, comme le soulignait André Ayçoberry, directeur du groupement VII (fibres de verre) chez Saint-Gobain : "en isolation au fond, ce que nous vendons ce n'est pas un poids, ce n'est pas un volume c'est une qualité d'isolation c'est-à-dire une économie de chaleur" [DIR 57, p. 159]. La demande soutenue en fibre de verre s'explique aussi par les nombreuses baraques militaires construites et utilisées lors de la Guerre d'Algérie [EXP 57, p. 21].

L'utilisation de la laine de verre se décline aussi sur une palette de produits standard et spécifiques intéressant plusieurs industries : “[...] les fabricants d'armoires frigorifiques ont été échantillonnés en fin d'année avec les produits TEL bakélisés et leurs premières réactions ont été favorables [EXP 57, p. 2]. La demande est en hausse chez Frigidaire qui, pour répondre à une demande croissant dès 1956, produit de plus en plus de réfrigérateurs nécessitant des laines isolantes [EXP 57, p. 21]. Ces besoins attestent donc de l'évolution de la consommation des ménages. Le schéma ci-dessous montre ainsi l'augmentation, dès les années 1950, en France, du taux d'équipement en réfrigérateurs. Dans les années 1960, c'est le bien électroménager le plus diffusé dans les ménages français.

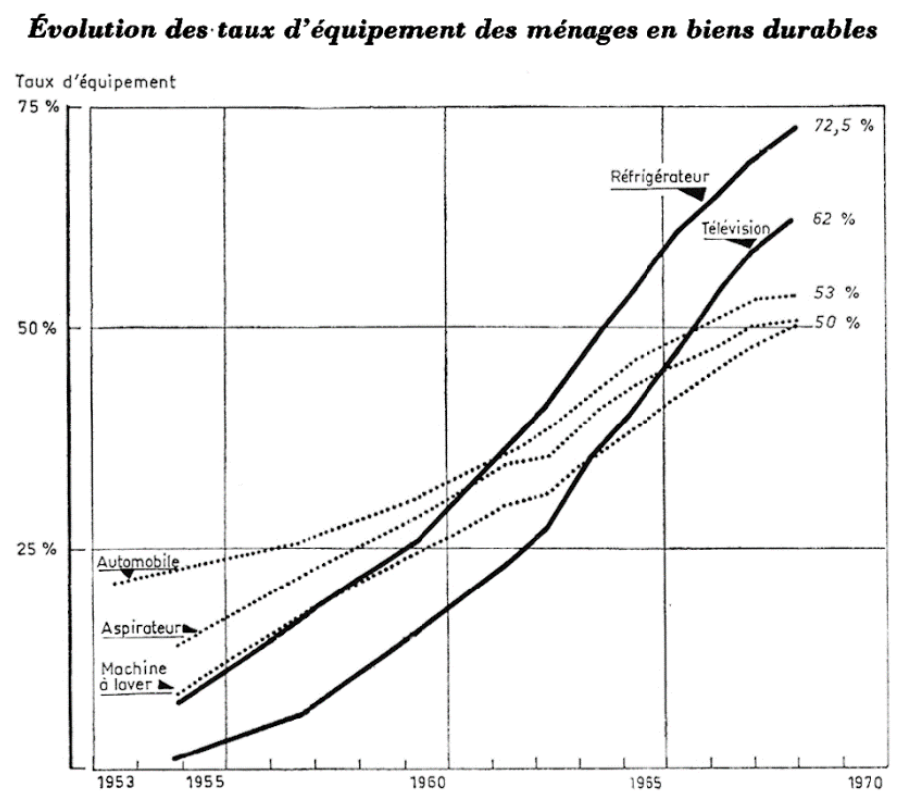

Figure. 1. Evolution des taux d'équipement des ménages en biens durables [LER 69, p. 65]

La genèse du TEL est aussi guidée par une forte volonté de prendre en compte les préoccupations des usagers comme le souligne André Ayçoberry : «En résumé, je dirais que la machine TEL a permis de supprimer le reproche essentiel qui était fait à la fibre de verre qui était celui de piquer» [DIR 57, p. 151]. La pose de la laine piquante occasionnait des blessures aux ouvriers et impliquait une augmentation de salaires de $25 \%$, donc des coûts supplémentaires pour les entreprises consommatrices. Le "toucher doux" [DIR 57, p. 154] est selon Lucien Deschamps (le directeur technique d'ISOVER à cette époque) l'objet de toutes les attentions des ingénieurs de Saint Gobain impliqués dans la genèse du TEL tels que Ivan Peyches, Pierre Heymes, Marcel Levecque, etc. L'utilisation de la bakélite nécessaire à l'encollage de la laine de verre vient ruiner ce toucher doux en donnant au produit final une certaine dureté. En effet, la bakélite brûle et fait disparaître l'huile indispensable à la douceur [DIR 57].

La publicité ci-dessous de 1954 [ISO 07, p. 91] montre que la laine de verre proposée tient compte des besoins des usagers. ISOVER s'appuie sur le gaspillage (d'argent) comme un argument pour promouvoir sa fibre de verre, plus économe que le charbon. La publicité insiste aussi sur la facilité de la pose qui était l'un des atouts majeurs de la laine de verre fabriquée avec le procédé 
TEL. Si faire un lien entre ISOVER et la problématique du développement durable est anachronique, on voit bien comment l'entreprise, ici Saint Gobain, assigne un sens à cette innovation du point de vue de l'usager.

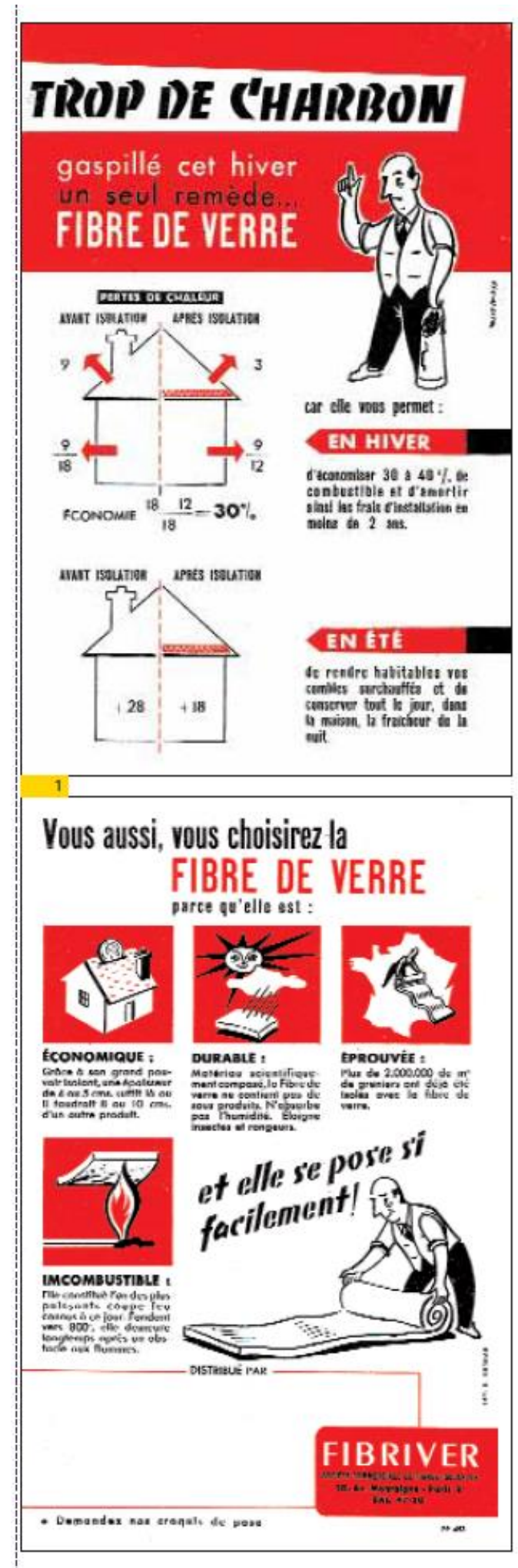

Illustration 2. Publicité pour la laine de verre en 1954 [ISO 07, p. 91].

En interne, les archives de Saint Gobain témoignent de questionnements relatifs aux usagers. Deschamps constate que la résistance au changement inquiète les cadres de l'entreprise : «Elle [la clientèle Hager] a ses habitudes et on peut se demander si elle acceptera facilement un produit nouveau sans doute intrinsèquement supérieur mais différent » [DIR 57].

\subsection{TEL ne naît pas dans un désert technologique}


Si le TEL répond à de nouveaux besoins et préoccupations pour les usagers, il n'émerge pas ex nihilo. Dès -3500 sur les bords du Nil, les Égyptiens faisaient chauffer du verre dans un bol, ils plongeaient ensuite un ferret (tige métallique) dans le verre fondu et d'un grand mouvement du bras, ils étiraient un fil grossier qu'ils enroulaient autour d'une forme en argile. En passant au four, ledit fil fondait, recouvrant alors intégralement la forme. Il ne restait plus qu'à casser l'argile pour avoir des objets creux en verre.

Si le verre filé tombe peu à peu dans l'oubli, il faut attendre la Renaissance pour qu'il refasse son apparition à Murano. Un premier ouvrier plonge un ferret dans du verre en fusion, un second colle sa tige et les deux partent en courant le plus vite possible, ce qui crée un fil. Ce procédé reste clairement artisanal. Le 7 avril 1919 est déposé le premier brevet relatif au "procédé Gossler". Ce procédé, qui donne naissance en 1920 à la firme Gossler à Hambourg, est inspiré du principe du rouet. Concrètement, le verre est fondu dans le four dont la partie inférieure est munie d'une filière, c'est-à-dire d'un récipient dont le fond est percé de multiples orifices. Des trous de cette filière, le verre liquide s'écoule. Cet écoulement forme des filets primaires donnant chacun naissance à une fibre unique qui vient s'enrouler sur le tambour roulant en continu. Ce procédé d'étirage mécanique fonctionnait à la vitesse de 13 mètres/sec. Il permettait de produire des fibres avoisinant les 25 microns et une production de $150 \mathrm{~kg} / \mathrm{J}$.
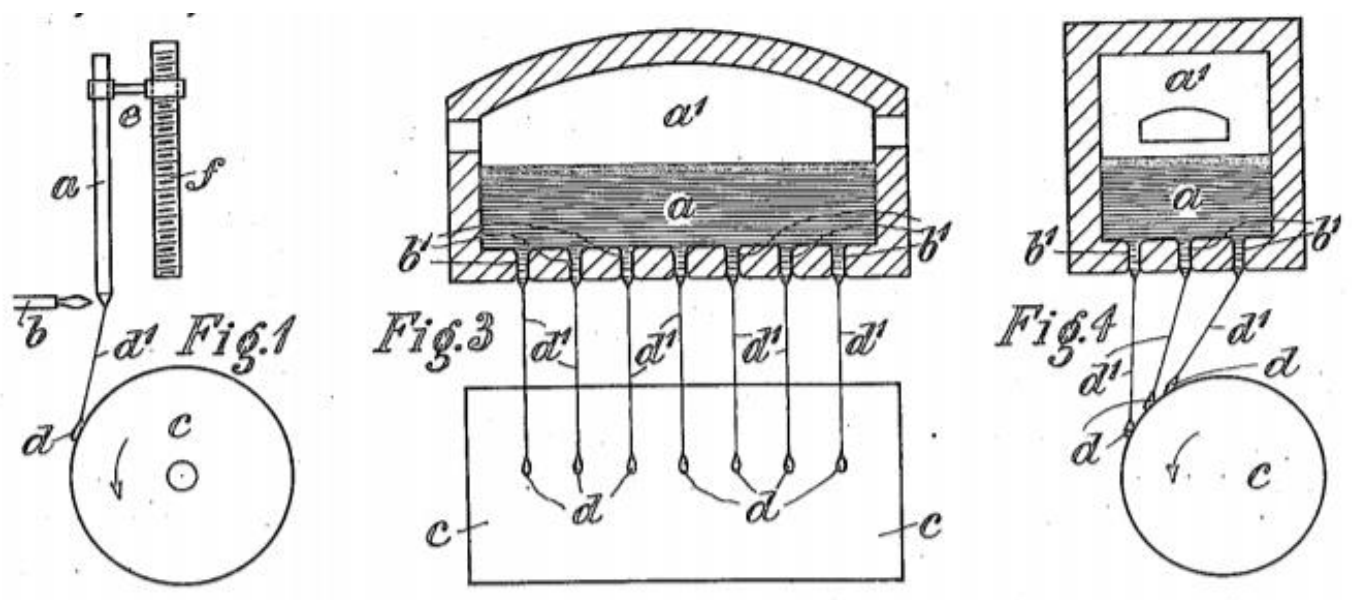

Schéma 1. Principe du procédé Gossler d'étirage de fibre de verre (Brevet US 1427014 du 22 août 1922 et Brevet procédé et appareil pour filer le verre FR 512925 délivré le 26 octobre 1920).

En 1928, Friedrich Rosengarth (1885-1977) invente le procédé dit “procédé Hager". Ce procédé serait né après que son inventeur aurait vu fonctionner une machine à barbe à papa. Il s'agit donc d'un procédé de fibrage centrifuge. Concrètement, on laisse couler par une tuyère un filet de verre fondu sur la surface d'un disque rainuré en réfractaire chauffé (appelé aussi fromage). Ce disque tourne à 3000/4000 tours/minute à quelques centimètres en-dessous de la tuyère. La force centrifuge chasse le verre vers la périphérie du disque. Le verre s'étire, par jet d'air, en filaments. Le procédé Hager permettra d'atteindre une production de 3 tonnes par jour [COM 57, p.4]. 


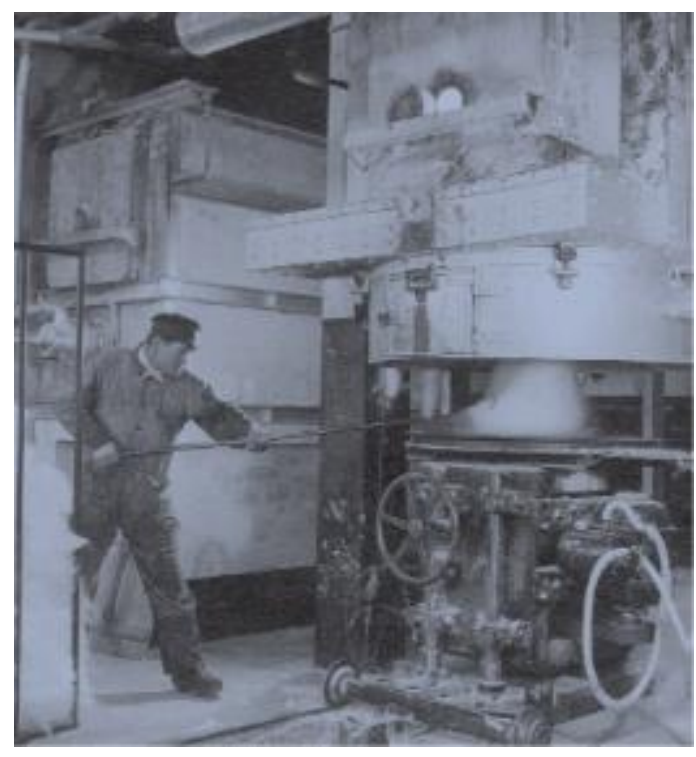

Illustration 3. Photographie du procédé Hager [ISO 07, p. 16]

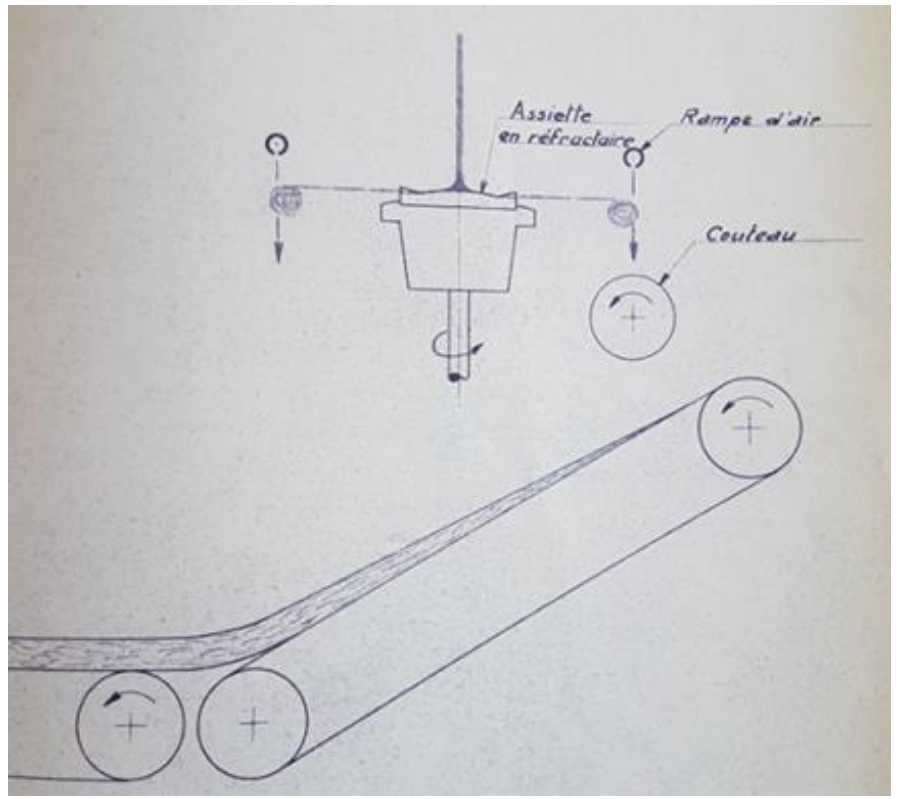

Schéma 3. Schéma de principe du procédé Hager [COM 57]

Entre 1928 et 1932, Games Slayter (1896-1964), ingénieur de la firme Owens Illinois développe le procédé Owens. C'est un procédé d'étirage par fluide pour lequel Slayter dépose, le 26 décembre 1933, une demande de brevet accordé le 11 octobre 1938 (brevet US 2133 236). Dans le procédé Owens, les filets de verre en fusion qui s'écoulent de filières en platine sont étirés sous l'action d'un jet de vapeur d'eau (10 à $\left.15 \mathrm{~kg} / \mathrm{cm}^{2}\right)$. Chaque filet donne naissance à un grand nombre de fibres de 12 à 14 microns. Le procédé Owens permet de produire une fibre moins piquante et plus régulière que celle obtenue par le procédé Hager. Devenu, en 1938, vice-président de la recherche et développement de la firme rebaptisée Owens-Corning Fiberglass Corp. (OCF), Slayter assure l'amélioration et la diffusion de son invention. 


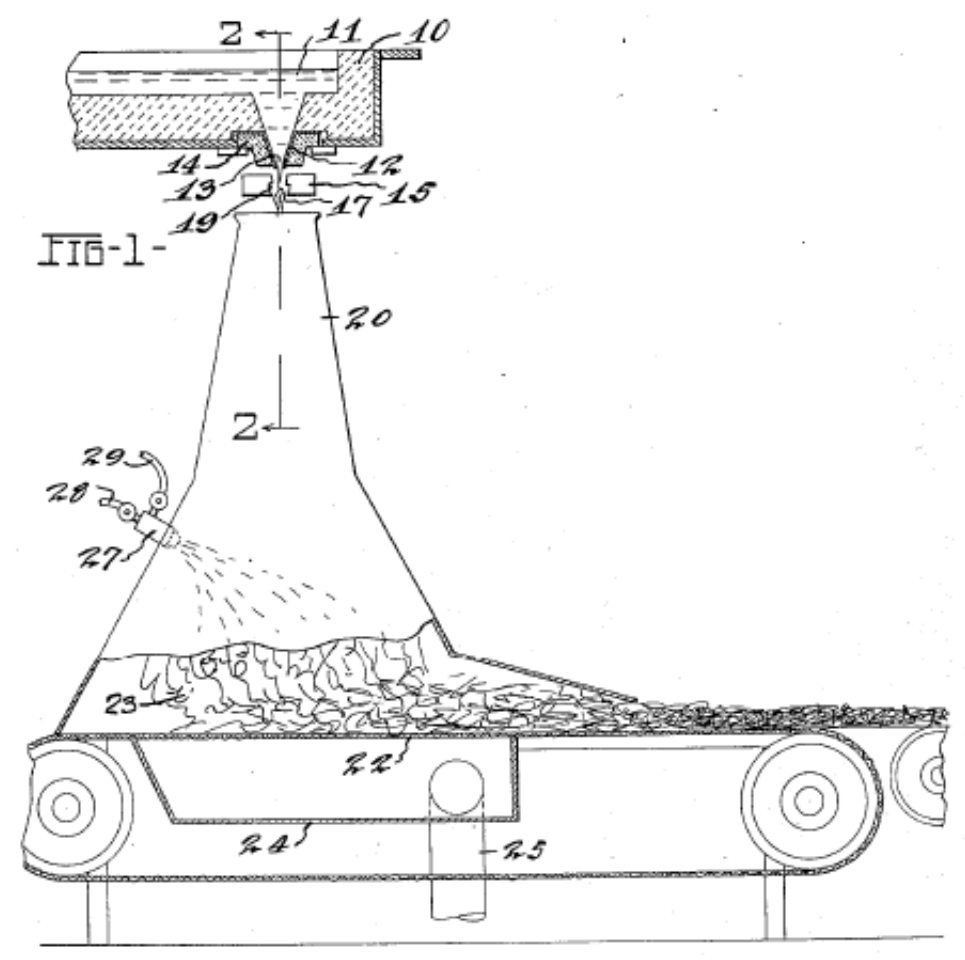

Schéma 4. Schéma de principe du procédé Owens [Brevet US 2206058]

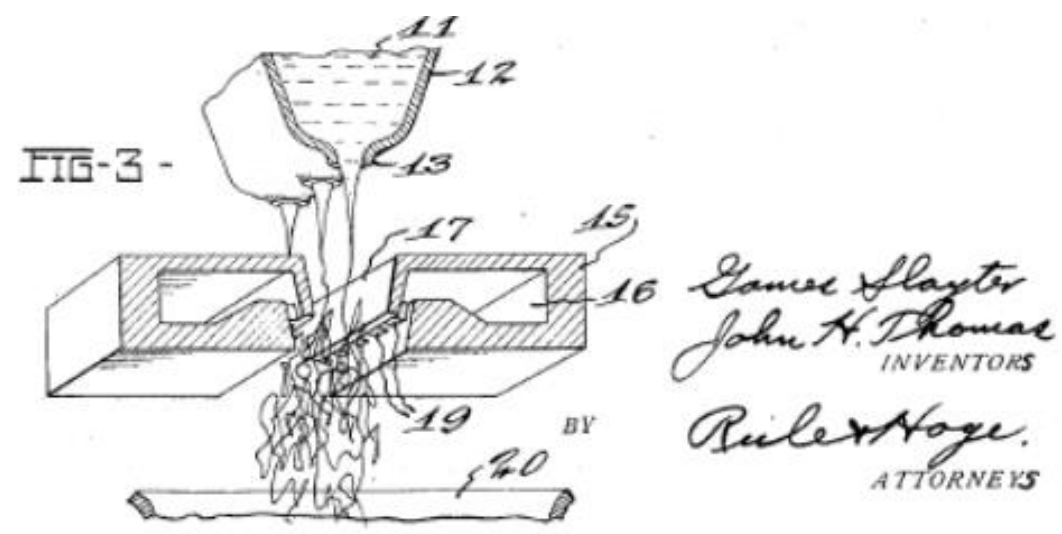

Schéma 5. Schéma de principe du procédé Owens (partie supérieure) [Brevet US 2206058]

Le TEL est, comme nous le verrons dans ce qui suit, l'héritier des procédés Hager et Owen. Il en reprend les caractéristiques pour à la fois s'en inspirer et s'en détacher.

\subsection{D'une stratégie d'acquisition de licences à l'avènement d'un nouvel industriel de I'isolation}

A l'époque, Saint-Gobain, peu convaincue par la fibre de verre, se situe dans une stratégie d'acquisition de licences dans ce domaine. L'année 1929 est ainsi marquée par la création de la société "La soie de verre" à Soissons, qui acquiert la licence Gossler pour la France. Isolant d'une exceptionnelle résistance, cette fibre est appelée "soie de verre". Elle était utilisée quasi exclusivement pour le calorifugeage des chaudières et tuyauteries à bord des navires militaires et marchands. Une de ses plus célèbres réalisations fut le calorifugeage des 29 chaudières du prestigieux paquebot "Normandie" dont la traversée inaugurale date du 29 mai 1935. 


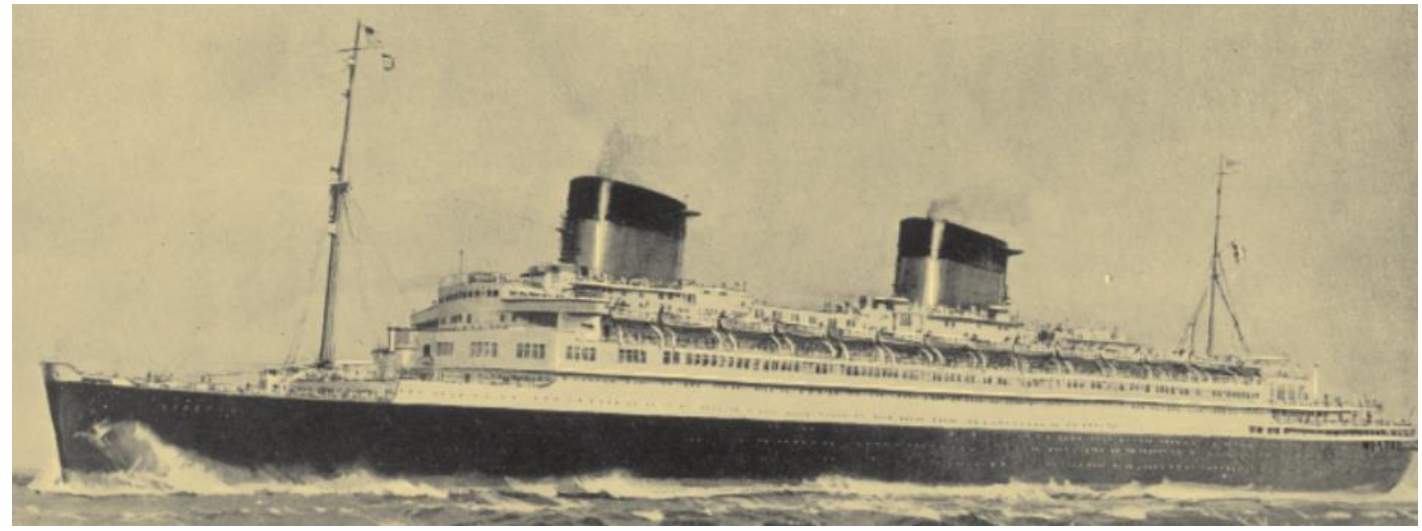

Illustration 4. Le Paquebot Liberté isolé avec 35 tonnes de laine de verre [ISO 07, p. 23]

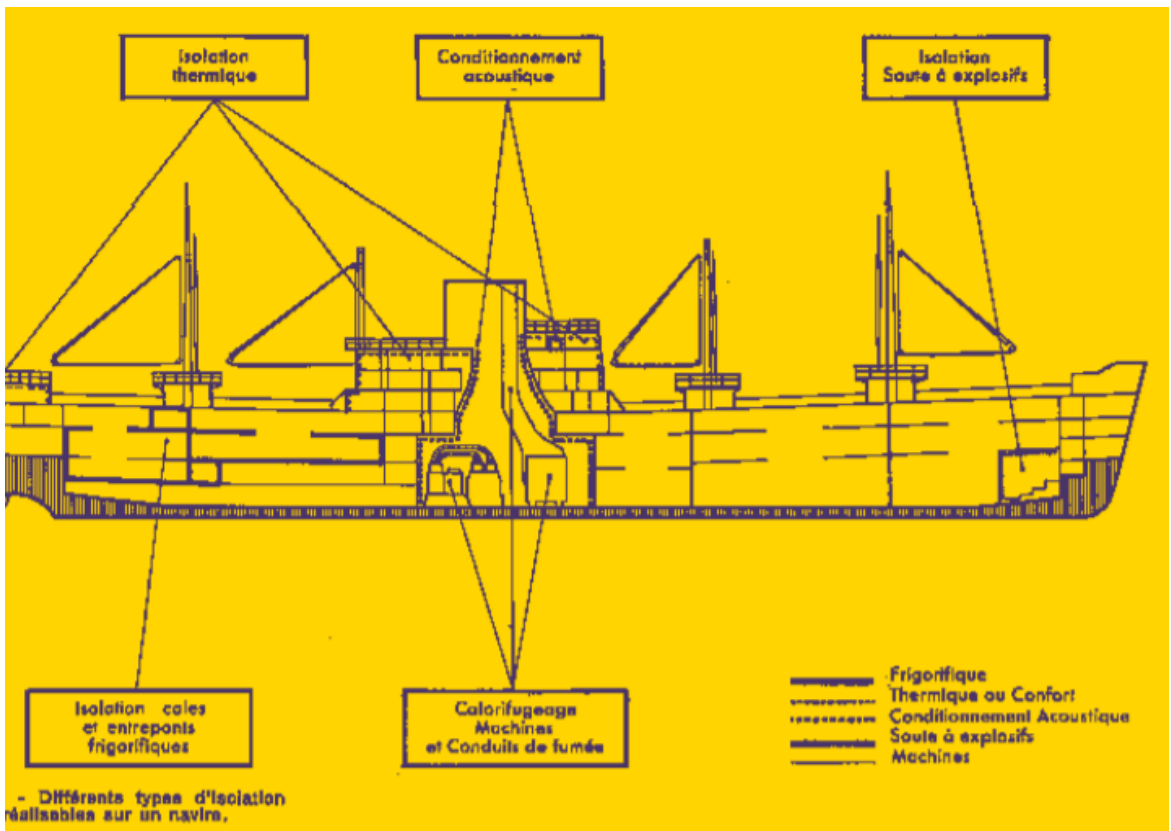

Illustration 5. Les types d'isolation sur un navire [ISO 07, p. 23]

De même, en 1930, la société néerlandaise Maatschappij Tot Beheer en Exploitatie van octrooien, filiale à $50 \%$ de Saint Gobain et à 50\% de la société Bicheroux, est chargée de l'exploitation de brevets et distribue les licences Hager. En 1933 Saint-Gobain refuse de devenir licenciée pour la France de Hager ; c'est la société des Glaces de Soissons qui devient licenciée de Hager pour 15 ans. Les premiers pas de Saint Gobain comme industriel de l'isolation datent de 1935, année durant laquelle l'entreprise rachète 50\% de la Glaswatte fondée par Hager en 1931. Ce rachat s'explique par le fait que le principe de la centrifugation semble intéressant et que le redoutable concurrent - le groupe Heye qui exploite déjà le procédé Owens - a déjà fait une offre à Hager.

En 1937, la Maatschappij signe un accord avec Owens Illinois après 3 ans de négociation. L'accord prévoit la «licence du procédé Hager pour Owens contre la licence du procédé Owens pour Saint-Gobain » [ISO 07, p. 41]. La création du "Pool” entre Saint-Gobain et Owens Illinois (sorte de coopération qui organise l'échange de toutes leurs informations sur le procédé Hager contre celles du procédé Owens et un accord sur les restrictions d'exportation) permet à la Maatschappij d'avoir les droits sur le procédé Owens, à l'exception de ceux acquis par Heye pour l'Allemagne, la Hollande, la Belgique et l'Italie.

En 1937, Saint-Gobain fonde ISOVER à Rantigny. Ainsi, le verrier français a accès à tous les procédés et devient un acteur de premier plan en produisant les 2/3 des 1500 à 2000 tonnes de fibres 
européennes (à l'époque les Etats-Unis produisent 20 à 25000 tonnes). Cependant, jusqu'en 1941, Saint Gobain se contente d'améliorer pas à pas les procédés qu'elle exploite, sans chercher à comprendre ils fonctionnent ou à innover en la matière. La Seconde Guerre mondiale va toutefois marquer un tournant majeur. Le conflit coupe Saint-Gobain de ses relations avec OCF au moment où le platine utilisé dans les filières du procédé Owens devient rare et que s'ouvre le marché de l'isolation. Tony Perrin (1877-1965) et André Ayçoberry, qui assumaient alors l'intérim de la verrerie, décident de lancer une activité de recherche sur le filage propre à Saint-Gobain et chargent Ivan Peyches (1906-1978) d'étudier et de critiquer les procédés existants afin de dégager des voies d'amélioration. Ce dernier, alors directeur du Laboratoire d'Essais Thermiques (LET), consacre alors une partie de son activité à cette question. Débute alors l'épopée du TEL.

\section{Un processus et des hommes}

\subsection{Un processus complexe et laborieux}

La genèse du TEL s'apparente à une épopée marquée par trois épisode, chacune d'elle se matérialisant par une évolution majeure du concept.

\subsubsection{Un processus de conception initié au cours de la première période (1941-1945)}

La première période, de 1941 à 1945 [PEY 77], est celle du LET, concept résultant d'une synthèse ingénieuse de l'approche allemande, qui associe la mécanique à la centrifugation (procédés Gossler et Hager), et de la méthode américaine du soufflage (procédé Owens). Le LET reprend le principe de la centrifugation alors jugé le meilleur par Ivan Peyches. En effet, ce procédé accepte le verre de récupération, ce qui est un atout en période de pénurie. Le LET se développe en substituant au disque rainuré réfractaire, dont les rainures s'usent rapidement, une assiette (la "tournette"). Cette substitution s'explique par le fait que les tentatives de modifications de matériaux effectuées sur le disque par Roger Lambert (dates naissance) en laboratoire s'étaient avérées infructueuses. Le verre collant mal sur ces matériaux, il donnait des fibres grossières. La "tournette", qui supporte des vitesses de rotation de plusieurs milliers de tours par minute sans se déformer et qui peut recevoir du verre en fusion à plus de mille degrés celsius, caractérise l'originalité du procédé de Saint-Gobain. Parce que constituée en Nicral (Nickel/Chrome/Aluminium), il faut utiliser du verre tendre pour préserver l'assiette. La "tournette" ressemble à une sorte de soucoupe au rebord percé de trous qui reçoit des billes de verre doux injectées du dessous par de l'air comprimé.

$\mathrm{La}$ "tournette" résulte d'un travail théorique et expérimental sur la centrifugation du verre orchestré par Ivan Peyches de 1941 à 1943. Ce dernier réalise des études aérodynamiques permettant de définir la forme idéale de l'assiette (du disque à des formes elliptiques). Un brevet demandé le 20 mai 1943 est délivré en France le 24 janvier 1951 sous la référence FR 981965. Ce dernier rappelle que grâce à la force centrifuge, le liquide est projeté hors des orifices sous formes de filets qui subissent ensuite, sous l'action de cette même force, un effet d'étirage qui les transforme en fils. L'air ambiant provoquant le refroidissement du verre, ce dernier finit par se solidifier et l'étirage ne peut plus se poursuivre. Partant du principe que plus on projette loin les filets plus l'étirage sera grand, la solution présentée dans le brevet porte donc sur la forme de l'assiette. Le choix de disposer les orifices de distribution dans l'arête est aussi guidé par le fait qu'en se solidifiant à proximité du corps du centrifugeur, les fibres tendent à engorger ce dernier et par suite à en empêcher le bon fonctionnement. 

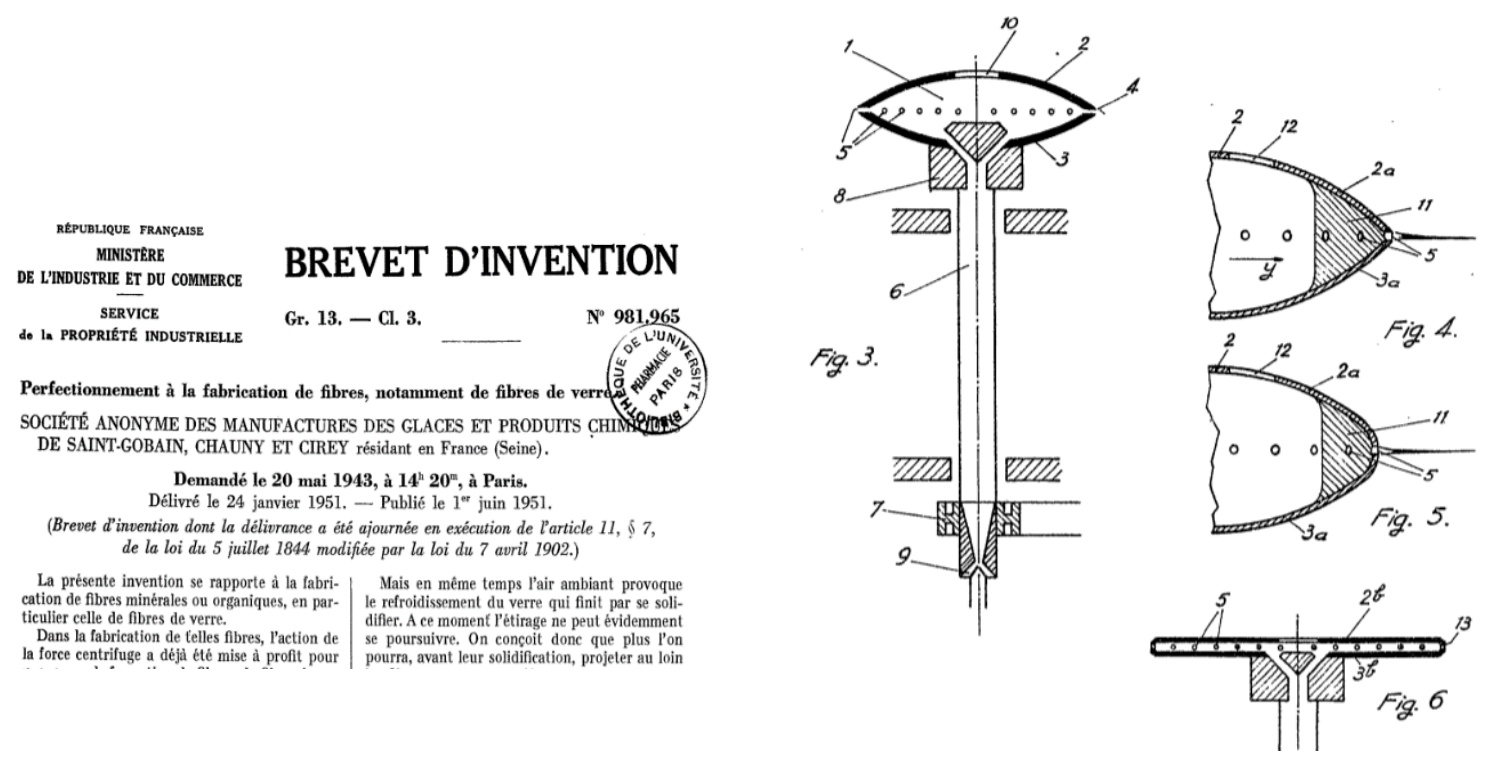

Schéma 6. Brevet d'invention relatif à l'assiette [Brevet FR 981965]

Le concept du LET associe à la centrifugation l'étirage des fibres par un gaz chaud, ce qui le distingue du procédé Owens qui étire la fibre à l'air ou à la vapeur. Au cours de cette première période, la réflexion porte sur l'amélioration de l'étirage en jouant sur la température. La solution imaginée consiste à d'abord à donner au verre, avant sa sortie, une température suffisamment basse $\left(700^{\circ}\right)$ pour que, du fait de sa viscosité, le verre sorte à une vitesse inférieure à celle qu'il prendrait du seul fait de la force centrifuge et soit peu apte à être étiré efficacement. Il s'agit alors d'élever la température $\left(900^{\circ}\right)$ du filet de verre après sa sortie de l'orifice pour qu'il puisse être utilement soumis à une action d'étirage, puis interrompre ce chauffage après un court parcours du filet. Cette invention donna lieu à des brevets, à l'instar du brevet demandé le 19 août 1943 et délivré le 14 février 1951 [Brevet FR 983422] (Schéma 7).

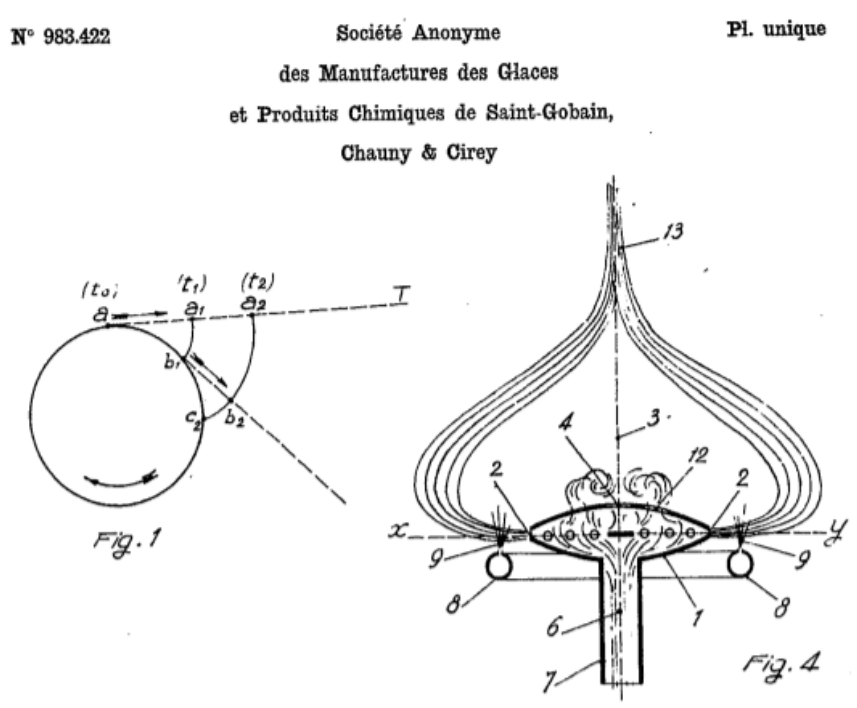

Schéma 7. Procédé et appareil pour la fabrication des fibres, notamment de fibres de verre [Brevet FR 983422]

L'étude de la première période de l'épopée du LET conduit à constater que la genèse de ce procédé innovant est marquée par un processus de conception utilisant une véritable approche expérimentale et dont le déroulé ne ressemble en rien à un long fleuve tranquille. Comme le souligne Raymond Villain 
«c'était passionnant ... Pour autant tout n'était pas rose, loin s'en faut. Le procédé en était encore à son balbutiement et beaucoup de chose ne fonctionnait pas encore... C'était parfois risqué : j'ai vu des assiettes de fibrage me passer au-dessus de la tête comme des soucoupes volantes » [VIL 05, p. 2].

Le surétirage posa de nombreux soucis. Si le fonctionnement de la première machine était correct à froid, à chaud l'assiette se déformait, ce qui produisait "quelques étincelles" [PEY 77]. Il faut attendre mai 1944 pour que le principe du surétirage se montre efficace et permette de produire des fibres passant de 25 microns à 16 microns.

Cette première période est aussi marquée par le besoin de développer de nouvelles connaissances. "A l'époque, les inventeurs et exploitants en l'absence d'une théorie des phénomènes, étaient contraints à n'avancer qu'empiriquement. Ils faisaient de la fibre et c'était l'essentiel. Mais pour le scientifique qu'était Ivan Peyches, il fallait savoir POURQUOI et COMMENT cela marchait" [BAT 06, p. 54]. Prenant acte du manque de connaissances sur les conditions physiques dans lesquelles étaient obtenues les différentes sortes de fibres, Ivan Peyches recrute Pierre Heymes en 1942 [ISO 07, p.68] pour mener une thèse sur la théorie du fibrage. Cette thèse permet de comprendre que la force d'étirage qu'un fluide peut exercer sur un fil fin (en l'occurrence le verre fondu) dépend à la fois de sa force et sa température. On est ainsi conduit à considérer que la relation de la recherche à l'innovation ne saurait se réduire à une simple logique d'application de connaissances scientifiques préalables, mais se nourrissent l'une l'autre: la recherche ouvre l'univers des possibles à l'innovation, de même que cette dernière ouvre celui des possibles de la recherche [FOR, 17].

\subsection{2. ... suivi d'une première reconception (1945-1952)...}

Si la première période de l'épopée du LET permet d'obtenir des fibres de bonne qualité, en revanche le débit est faible et le toucher des fibres demeure irritant. C'est dans ce contexte qu'émerge l'idée de retourner l'assiette de fibrage. Le LET devient le TEL, par un retournement physique autant qu'acronymique. S'engage alors, de 1945 à 1952, une reconception du procédé de fibrage. Dans le nouveau procédé obtenu, le verre arrive par le haut. La réception s'en trouve alors facilitée. Au cours de cette deuxième période de l'épopée du LET, Saint-Gobain retravaille à la fois la composition du verre, le brûleur externe et l'assiette. La forme, le matériau et le nombre de trous feront l'objet de recherches expérimentales et mises au point nombreuses que l'on retrouve dans les cahiers techniques.

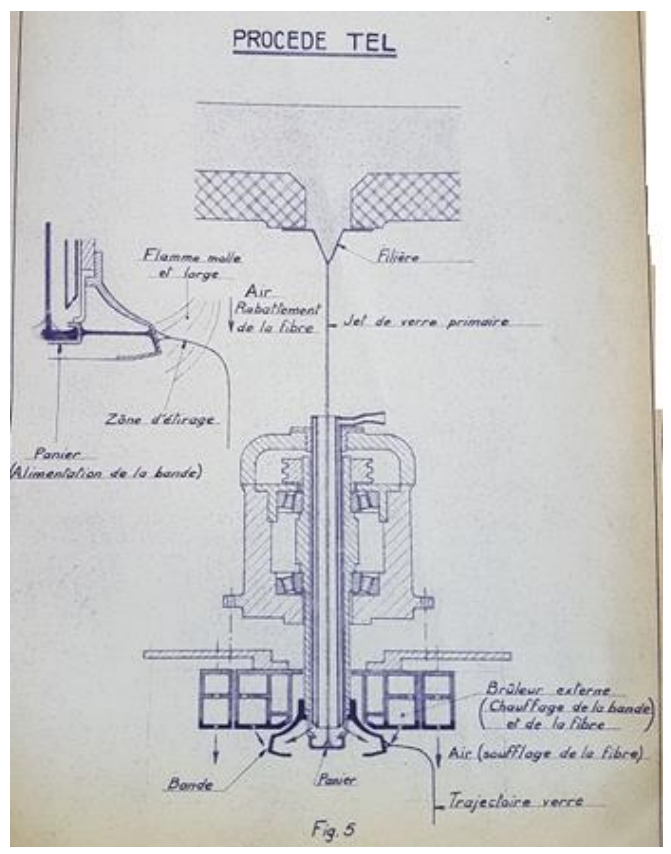

Schéma 8. Procédé TEL [COM 1957] 
Malgré des essais réalisés dès 1948, le procédé TEL n'est pas au point. La durée de vie de l'assiette est trop courte. Du fait de l'absence d'aciers spéciaux résistants à la haute température du verre en fusion entre 1400 et $1500^{\circ}$, les verres maintenus à une température trop basse dévitrifient et obturent les trous. Le débit est alors trop faible et le procédé a une capacité de seulement 4 tonnes par jour de laine de verre. Les fibres de verre sont trop épaisses puisque leur diamètre atteint 8 microns . Ces fibres sont par ailleurs peu efficaces car trop peu d'air est enfermé entre elles. On entre alors dans la troisième période, celle du Supertel (1952-1957).

\subsection{3. ... et d'une seconde reconception (1952-1957)}

Le nom de Supertel remonte au 13 octobre 1952 [ISO 07 p.72], date à laquelle il est décidé de consacrer des efforts de recherche pour appliquer le principe de fibrage pensé par Pierre Heymes à la machine TEL, et ce sous la supervision de Marcel Lévecque (1922-1979). Le Supertel améliorera donc le procédé rotatif [Aufaure in TOL 80, p. 6].

Le principe imaginé par Pierre Heymes émerge bien avant cette troisième période ; il résulte d'une visite d'une exposition des premiers missiles V1 et V2 : "Il [HEYMES] chercha comment produire de tels jets de fluide et eu la solution en visitant avec PEYCHES en octobre 1944 dans un hangar derrière Notre-Dame, une exposition de V2, récupérés non explosés, par la Défense Passive »[BAT 06, p. 63]. Il comprend alors comment, dans le V2, une combustion explosive entretenue dans l'enceinte semi-close permet d'éjecter des gaz très chauds à très grande vitesse. Par analogie, Pierre Heymes transfère ce principe de solution pour étirer les fibres. Cette idée donne ensuite naissance à plusieurs brevets. Le premier, demandé le 12 février 1945 et délivré 14 août 1951 [Brevet FR 994865 A], présente un procédé d'étirage effectué par des courants gazeux se déplaçant à grande vitesse et entraînant la matière à étirer. Ce procédé permet d'obtenir des fils très fins. La combustion de mélange gazeux dans l'enceinte peut produire une température de 1200 degrés.

RÉsumé.
L'invention vise :
$1^{\circ}$ Un procédé pour l'étirage, sous forme de fibres,
de matières thermoplastiques, telles que le verre,
portées à haute température, consistant à effectuer
l'entraînement de la matière à étirer, au moyen de
la détente, à travers un orifice, d'un fluide à tempé-
rature élevée, résultant d'une combustion produite
préalablement dans une enceinte communiquant avec
l'orifice de détente.
$2^{\circ}$ Dans la réalisation de ce procédé suivant $1^{\circ}$,
les dispositions suivantes appliquées isolément ou en
combinaison:
a. Les gaz de combustion sont produits par l'in-
troduction dans l'enceinte d'un élément comburant,
par exemple un gaz, et d'un combustible gazeux;
$b$. Le combustible est introduit dans l'enceinte sous
forme liquide;
c. Le combustible est introduit sous forme solide;
d. La combustion dans l'enceinte est provoquée par
inflammation au début du travail;

[994.865]

$e$. La combustion est provoquée par une inflammation entretenue par exemple par allumage électrique;

$f$. Les amenées de combustible et de comburant sont disposées de manière que la combustion dans l'enceinte soit localisée au voisinage de l'orifice de détente;

g. Le fluide d'étirage agit sur la matière à étirer pendant le passage de ce fluide au travers de l'orifice de détente;

$h$. La matière est introduite dans l'enceinte où a lieu la combustion;

$i$. L'entrée en contact de la matière à étirer avec le fluide d'étirage a lieu après que le fluide est sorti de l'orifice de détente.

$$
\begin{aligned}
& \text { SOCIÉTÉ ANONYME DES MANUFACTURES } \\
& \text { DES GLACES ET PRODUITS CHIMIQUES } \\
& \text { DE SAINT-GOBAIN, CHAUNY \& CIREY. } \\
& \text { Per procuration : } \\
& \text { ARMENGAUd aîné. }
\end{aligned}
$$

Illustration 6. Revendication du brevet de 1945 [Brevet FR 994865 A]

Une première addition à ce brevet est demandée le 31 mai 1946. Elle est accordée le 25 juin 1952 en France, puis le 13 janvier 1953 aux Etats-Unis. Elle présente une invention qui articule l'effet d'étirage par les gaz produits dans la chambre à combustion (l'invention du Brevet principal du 12 
février 1945) et la disposition du corps centrifugeur (qui a lui-même fait l'objet d'une demande de brevet le 20 mai 1943).

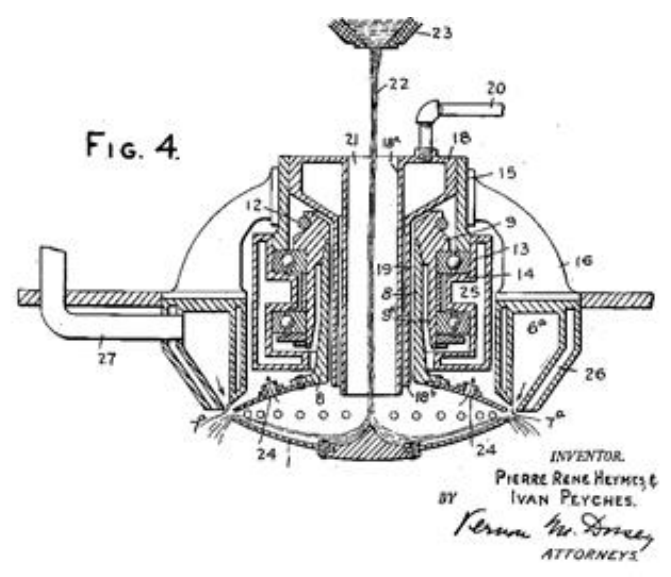

Schéma 9. Mosaïque du brevet d'invention du 31 mai 1946 [Brevet US 2624912 A]

Comme on peut le constate sur le schéma ci-dessous, le Supertel est clairement l'héritier du travail de Pierre Heymes.

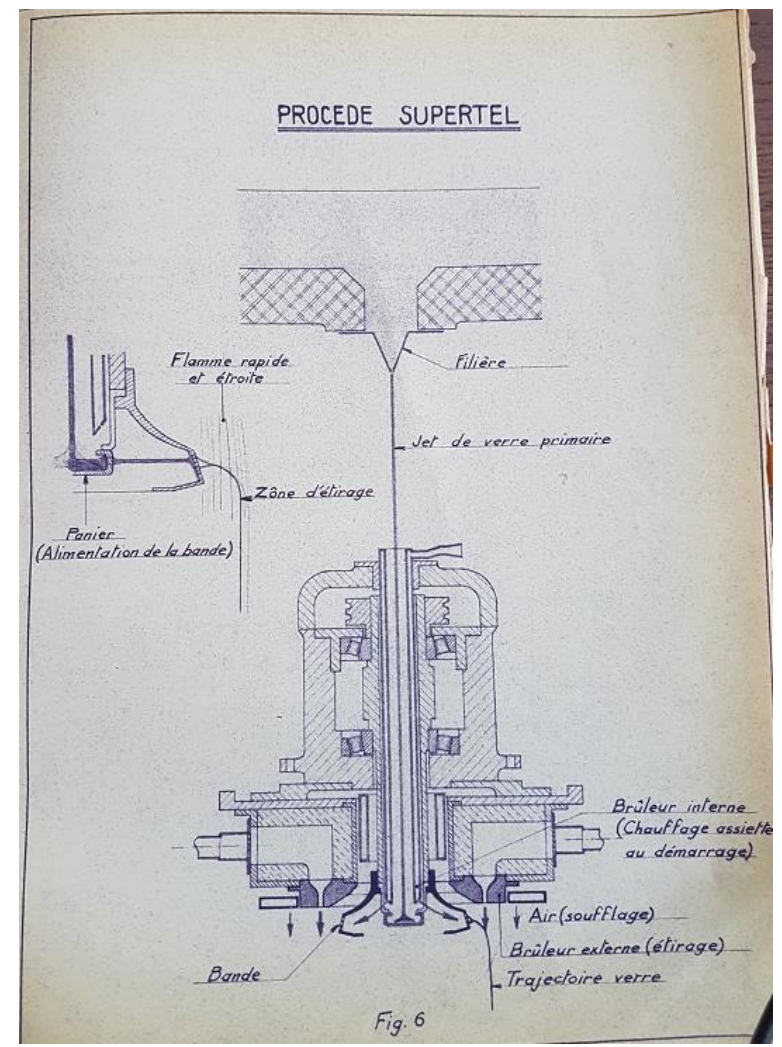

Schéma 10. Procédé SUPERTEL [COM 57, non paginé]

Au cours de cette troisième période de l'épopée du TEL, l'équipe de Marcel Levecque met au point les brûleurs, augmente la hauteur de la bande de fibrage, ce qui lui permet de loger un plus grand nombre de trous. Cette augmentation pose de nouveaux problèmes : comment remédier au problème de l'obtention d'une fibre de diamètre uniforme quand il y a plusieurs rangées de trous et de ce fait des conditions différentes derrière les orifices de projection (en épaisseur de couche de verre et en température) ? Les rangées du haut sont près de la flamme du brûleur et produisent des fibres fines. En revanche, parce que les rangées du bas sont frappées par une flamme moins rapide, les fibres sont plus grosses, plus longues. Si la laine obtenue semble à première vue plus belle [DIR 
57, p.155] son lambda ${ }^{3}$ est cependant moins bon. Le brevet demandé 28 février 1955, auquel ont contribué Marcel Levecque et René Piot, apportera une solution à ce problème via l'insertion d'un organe distributeur en forme de cône en alliage métallique présentant une forte mouillabilité.

Au final, le processus de conception-reconception du TEL aura duré plus de 15 ans, puisque la consécration définitive du nouveau procédé arrive en septembre 1957 lorsque le procédé Owens exploité à Rantigny fut arrêté au profit du Supertel. Ce processus est ponctué d'un flot continu d'inventions (on dénombre plus de 700 brevets) et de va et vient permanents avec la recherche : "la collaboration étroite de l'usine et de la recherche fut indéniablement à la base du succès. » [BUL 67, p.6]. Mais la genèse du TEL révèle aussi qu'il n'y a pas de dynamique de l'artefact sans reconception. La dynamique du TEL est due à des motifs internes, à savoir un besoin de perfectionnement visant à améliorer la qualité des fibres produites tout en réduisant les coûts de production.

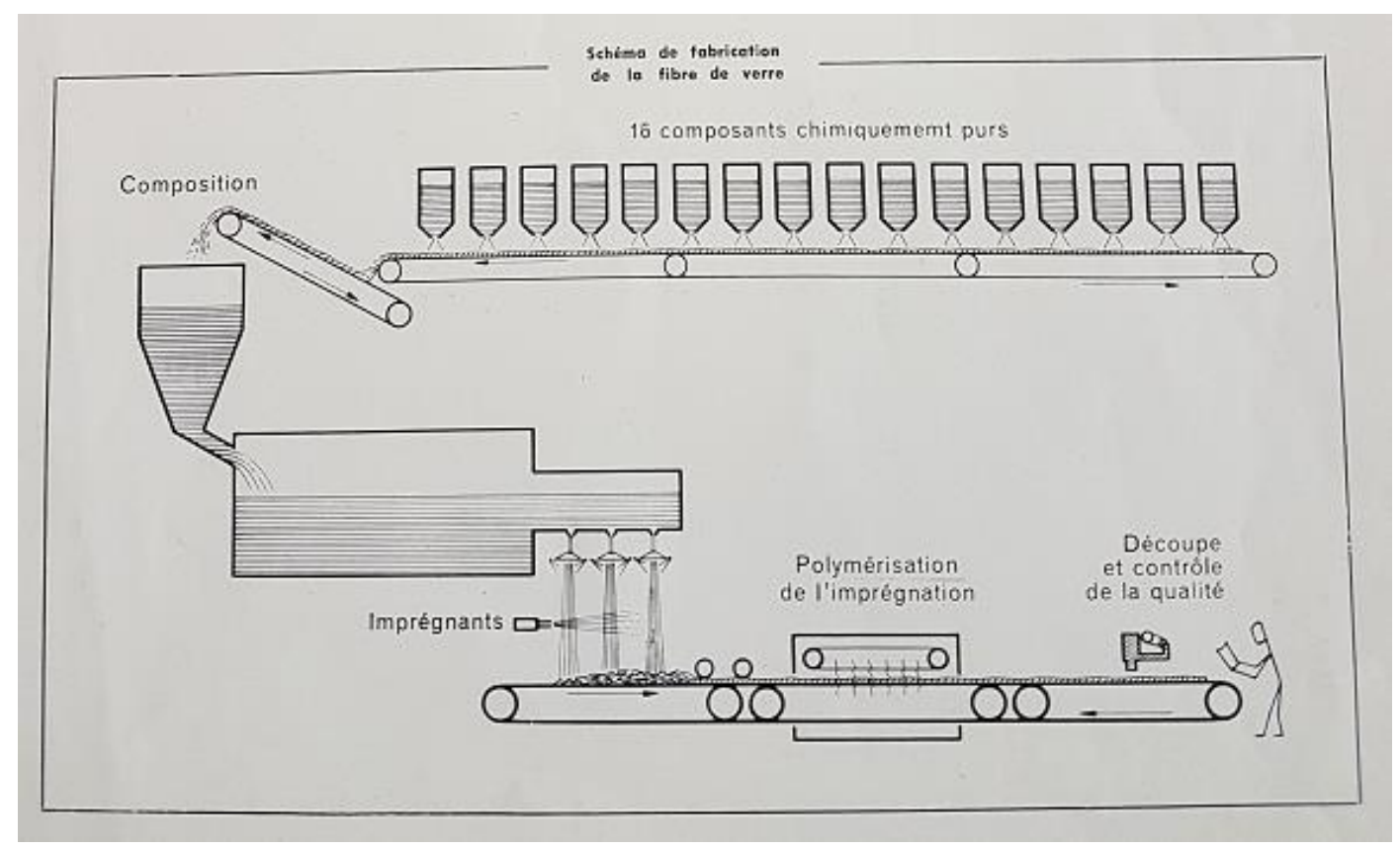

Schéma 11. Vue d'ensemble du procédé [BAI 59]

\subsection{Une œuvre collective qui s'appuie sur des personnalités}

Si le Supertel fait en soi figure d'innovation technologique importante, on ne saurait occulter le rôle qu'ont joué les acteurs au cours de sa genèse. Le procédé TEL a été porté par des personnalités hors normes, capables de bousculer la cartographie des savoir, de s'aventurer hors des sentiers battus, de faire des liens où il n'en existait pas, bref de déployer une forme de pensée qualifiée de « rationalité créative » et dont nous avons déjà indiqué qu'elle est à l'origine même de l'innovation [FOR 17a]. Elles ont également su créer des synergies témoignant du caractère collectif de l'aventure. De la sorte, cette biographie d'innovation déconstruit le mythe romantique du génie solitaire guidé par sa seule inspiration.

\subsubsection{Des personnalités hors du commun....}

L'histoire du TEL laisse entrevoir des acteurs incontournables sans qui ce procédé n'aurait certainement pas pu voir le jour. Les personnalités marquantes d'Ivan Peychès, de Pierre Heymes ou de Marcel Levecque sont souvent évoquées dans les ouvrages retraçant l'histoire du TEL ou les

\footnotetext{
${ }^{3}$ Le coefficient Lambda d'isolation thermique sert à exprimer la conductivité thermique d'un matériau isolant. Concrètement, le coefficient lambda d'un matériau correspond à la quantité de chaleur qu'un isolant thermique donné laisse passer en une seconde sur une surface d'un mètre carré et pour une différence de température équivalente à 1 degré entre les deux milieux isolés. Dit autrement, plus le lambda est faible, plus le matériau est isolant (conductivité faible).
} 
interviews que nous avons pu mener. Les discours attestent de leur capacité hors normes à se projeter dans un futur plus ou moins proche, à prendre des risques, à concevoir et à créer de nouveaux objet techniques.

A propos de Pierre Heymès, à l'origine du principe de fibrage, il est par exemple écrit dans les documents internes de Saint-Gobain : «Notons que, toujours visionnaire, il [Heymès] a dès cette époque proposé de la [l'assiette de fibrage] renverser, donc il a conçu le TEL sans le savoir » [ISO 07, p. 68]. Pierre Heymès apparaît comme étant pourvu de cette fine intuition qui lui permet d'identifier ce qui fournira la solution aux problèmes liés à la conception du TEL. L'intuition et la vision ne suffisent pas. Il possède d'autres qualités : qualifié de "meilleur de nos ingénieurs d'essais » [ISO 07, p. 68], le diplômé des Arts et Métiers est doté d'une capacité d'analyse plus importante que la normale. Pour ne donner qu'un exemple, il creuse la piste du fibrage par fluide et « comprend que la force d'étirage qu'un fluide peut exercer sur un fil fin, en l'occurrence un filet de verre fondu dépend à la fois de la vitesse et de sa température » [ISO 07, p. 68]. C'est à cette occasion qu'il reprend le principe d'éjection du gaz chaud à grande vitesse des chambres de combustion des V2 précédemment évoqué.

Ivan Peychès, qui fut directeur du projet LET, possède, quant à lui, l'aura d'un « grand maître verrier qui dirigeait le centre de recherches de Saint-Gobain situé à la Villette » [DAR 07, p.7]. Il sait s'entourer des personnes adéquates au projet qu'il développe. En outre, ce chercheur semble posséder un esprit vif. A plusieurs reprises, l'ouvrage retraçant la mise au point du TEL atteste d'un esprit sagace : «En quelques mois Ivan Peychès s'est fait son opinion : le procédé centrifuge Hager est le plus logique des trois [Gossler, Owens ou Hager] d'un point de vue physique » [ISO 07, p. 58]. Dans le même ordre d'idée, il est écrit que «Très vite, Ivan Peychès imagine de l'associer [l'étirage des filets de verre sous l'action d'un gaz de combustion à très haute vitesse] au fibrage centrifuge qu'il voit à l'œuvre au cours des essais TEL dont il a pris la responsabilité » [ISO 07, p. 72]. On comprend alors combien ce directeur du laboratoire est une personnalité hors norme : il possède de nombreuses connaissances, sait s'entourer et comprend vite [CHO 18].

Marcel Lévecque apparait comme une figure marquante de l'épopée du TEL. Nombreuses sont les citations qui mettent en avant cet esprit brillant qui a su faire du TEL un vrai procédé industriel. A l'heure de sa disparition en 1979, un hommage lui est rendu dans un livret intitulé "Marcel Lévecque notre ami” dans lequel ses collègues témoignent. On peut lire :

"Ses dons étaient multiples, son registre prodigieusement étendu. Comme un cristal aux mille facettes, il pouvait être tour à tour jovial, quelques fois truculent ou grave, non conformiste et pragmatique, artiste et homme d'action, cartésien dans ses raisonnements, mais rêveur quelque fois, en tous cas visionnaire dans sa perception du futur. Il avait un esprit critique et méthodique tout en étant un tantinet bohème". [Tolédano, 1980, p. 10].

Cette citation éclaire bien la personnalité complète de celui qu'on appelle "le patron" qui «s'intéresse à tous les aspects de la machine, brasse des idées à la pelle, accable ses collaborateurs de travail. A la fois "Théoricien" et "expérimentateur pragmatique et ingénieux» [ISO 07, p. 72], il possède également une large palette de connaissances. Il maîtrise à la fois les propriétés des verres classiques, celles des aciers à haute performance, ou encore les procédés de combustion [Aufaure in TOL 80]. Ces qualités ont été propices au développement de la rationalité créative. Ivan Peychès conjugue deux modes d'étirage : l'étirage par centrifugation et l'étirage par fluide. D'ailleurs selon Ivan Peychès, «C'est de rapprochements illégaux que naît la nouveauté » [PEY 57, p. 6]. C'est également parce qu'il use de sa rationalité créative que Pierre Heymès parvient à traverser les savoirs et importe le principe des chambres de combustion des V2 allemands dans le procédé de fibrage. Cet exemple, plus que tout autre montre, sa capacité d'observation indéniable et son aptitude à opérer des analogies de façon pertinente en allant chercher des connaissances d'un domaine à un autre. Pour sa part, Marcel Lévecque mobilise ses connaissances en aéronautique dont Aufaure souligne qu'elles «l'ont beaucoup aidéldéfinir de façon précise les procédés de contrôle de 
combustion qui n'étaient pas adaptés à l'époque à une telle technique de pointe» [Aufaure in Tolédano, 1980, p. 6]. Et ce dernier de préciser qu'il fallait « une imagination aiguë comme celle de Marcel pour tirer les conclusions. Pour rassembler les éléments épars de ces observations. Marcel savait être réceptif et saisir l'insaisissable » [Aufaure in Tolédano, 1980, p. 6]. Heymès apparaît capable de traverser les savoirs et de transgression aventureuse [FOR 17a] :

«Ces contradictions apparentes qui ont un pouvoir destructeur chez un être faible, incitaient en revanche une nature comme la sienne, douée d'une forte vitalité et d'une intelligence affûtée aux explorations audacieuses qui déclenchent l'impulsion créatrice et la renouvellent [...] pouvait se permettre de briser les habitudes, d'enjamber les barrières hiérarchiques, de nouer des liens nouveaux entre les choses, de créer des courants et de frôler des sommets inaccessibles aux êtres moins richement doués » [Tolédano, 1980, p. 12].

Pour autant, ces qualités hors du commun s'expriment, comme nous allons le voir dans ce qui suit, dans une œuvre collective.

\subsection{2. ... parties prenantes d'une œuvre collective}

Il faut une équipe solide, dotée de membres complémentaires, pour passer de l'idée à la pratique et mettre au point les procédés évoqués ci-dessus à l'aide d'une succession d'expériences : «on travaillait beaucoup, sans horaire, des nuits entières s'il le fallait » [BER 05].

« c'est grâce à la clairvoyance prémonitoire et à l'obstination d'hommes de grande classe Roger Lacharme, Lucien Deschamps et Gabriel Aufaure, grâce aussi à leur confiance inébranlable en Marcel Lévecque que les barrières du doute, du scepticisme et de la routine purent être abattues et que Marcel put disposer des moyens nécessaires pour mener à bien sa mission » [Tolédano, 1980, p. 20].

La préface de l'ouvrage célébrant les cinquante ans de la laine de verre est rédigée par Claude Imauven (alors directeur général adjoint de Saint-Gobain en 2007, année de la parution). Cette préface traduit bien l'aspect collectif de l'aventure du TEL:

«[...] mais ce procédé fruit du labeur d'ingénieurs et de techniciens s'est développé graduellement en utilisant une véritable approche expérimentale. A l'aventure des pionniers d'avant-guerre a donc succédé l'âge d'or des ingénieurs enthousiastes qui ont développé le TEL. » [ISO 07, p.11].

Jean Battigelli souligne que « [...] l'équipe (...) constituée de gens sortis du rang, dont la qualité la plus évidente, la complémentarité lui permettait de fonctionner en autonomie et d'avancer en bloc derrière le chef aimé qui savait où la mener » [BAT 06, p. 94].

Ces écrits viennent corroborer la thèse selon laquelle l'innovation se nourrit d'une intelligence collective guidée par des individus charismatiques, hors du commun, dotés d'un savoir technique et scientifique conséquent. Marcel Lévecque «a su aussi les guider et les orienter vers un but commun » [Aufaure in Tolédano, 1980, p. 6]. On est loin du mythe du "génie solitaire" [CAL 94, p. 6], [FOR 17b]. Nous suivons en cela la traduction ouverte par Michel Callon, qui nous invite à déconstruire ce mythe au profit d'une cartographie et d'une analyse fine des réseaux sous-jacents à la genèse de l'innovation. Si de grands innovateurs sont présents sur la scène, leur aura cache la réalité des collectifs sous-jacents :

«Edison, Steve Jobs: voilà autant de noms donnés à des collectifs comportant un grand nombre d'acteurs rassemblés en un même lieu et interagissant constamment pour mettre en forme l'innovation » [CAL 94, p. 6]. 
Dans le cas présent, si des figures comme Marcel Lévecque ou Ivan Peyches attirent la lumière à eux, leurs visions, leurs idées ont pu se concrétiser parce qu'une équipe et un environnement portaient les projets dans lesquels ils se sont engagés. Le mythe du génie solitaire est d'ailleurs pointé du doigt dans la préface de l'ouvrage publié au moment du cinquantenaire du TEL : «Cette innovation [le procédé TEL] élément d'une réussite industrielle exemplaire, n'est pas née soudainement du cerveau d'un génie solitaire » [ISO 07, p. 11].

\section{En guise de conclusion}

Faire la biographie d'une innovation permet de révéler la complexité du processus expliquant sa genèse. La biographie du TEL proposée dans ce court article nous a permis de constater que ce procédé n'est pas né ex nihilo. Il s'inscrit dans un environnement économique (les besoins d'isolation d'après-guerre), scientifique (état des connaissances sur le verre) et technique (on connaît des modes d'étirage du verre) rendant l'innovation possible. Evoquer un tel environnement permet de montrer en quoi l'innovation n'est pas neutre. La genèse du TEL n'est pas étrangère à une stratégie industrielle d'une grande entreprise, en l'occurrence le glissement d'une stratégie d'acquisition de licences de Saint-Gobain à la décision de devenir un acteur de l'isolation à part entière. La biographie du TEL permet aussi de mettre en perspective le procédé final en retraçant le processus de conception et reconception qui l'a vu naître, puis se développer. Cette vision processuelle nous pousse à ne plus seulement considérer une innovation de l'extérieur, comme un donné, mais comme une œuvre collective que l'on peut comprendre en cartographiant des acteurs clefs et en traçant l'ensemble des idées, des choix, etc., jalonnant sa genèse. Malgré son apparence descriptive, cette approche à la fois environnementale et processuelle est en outre riche d'enseignements. Elle permet notamment de déconstruire certains a priori concernant l'innovation.

Le premier enseignement est que la biographie du TEL réactualise la thèse artificialiste [FOR 03] selon laquelle la genèse de l'innovation ne peut être comprise qu'à partir d'une approche multi niveaux. L'approche macroscopique est centrée sur l'étude du contexte (ou environnement externe) dans lequel s'intègre l'innovation à mesure de son développement. C'est à ce niveau qu'est considéré l'environnement socio politique, le système d'artefact en place, l'état des connaissances scientifiques et techniques disponibles, etc. Parce qu'elle occulte la dynamique interne du processus d'innovation, l'approche macroscopique, éventuellement teintée d'un certain évolutionnisme, ne suffit pas en soi pour comprendre la genèse de nouveaux objets techniques. Elle doit être complétée par une approche mésoscopique, centrée sur le processus de conception. Quel a été le besoin identifié par les concepteurs clefs ? Comment ont-ils développer les solutions ? Quand ? En combien de temps ? En faisant quoi ? Etc. Enfin, pour observer et comprendre encore plus finement ce qu'est la conception, il faut descendre jusqu'au niveau microscopique. Ce niveau, centré sur les acteurs, permet de considérer les compétences, stratégies cognitives et type de rationalité mises en œuvre. C'est à ce niveau que s'exprime la rationalité créative, l'analogie, etc. Cette approche multiniveaux permet non seulement de s'émanciper d'une vision déterministe de la genèse de l'innovation, mais aussi de comprendre pourquoi innover n'est pas qu'affaire granduelle, mais aussi de cheminement incertain, de sauts, etc.

Le second enseignement de cet article réside dans le fait que l'approche biographique met en lumière les chaînes causales entre les niveaux d'analyse mentionnés ci-dessus. La première est celle poussée par les besoins. Si le procédé TEL s'est développé, c'est parce qu'il permet de répondre à la forte demande en isolation. Celle-ci provient d'abord du développement du transport de marchandises par navires dotés d'un moteur thermique, puis de la hausse des constructions d'habitations, en lien avec le Baby boom, les Trente Glorieuses et la société de consommation. Une seconde chaîne causale concerne les usages. Les propriétés de la laine de verre produite par le procédé TEL satisfont des exigences dérivées des usages, par exemple le confort du toucher, la facilité de la pose, etc. Mentionner les usages signifie aussi s'intéresser à la résistance au 
changement; toute innovation étant nouvelle par définition, son adoption et son appropriation génèrant certains coûts.

Le troisième enseignement porte sur la nature individuelle, voire subjective, et sociale du processus d'innovation. Les biographies d'innovation nous permettent de réhumaniser l'innovation et nous évitent de penser l'objet technique, le procédé TEL dans notre cas, comme une chose se mouvant dans un désert culturel, social, etc. Considérer la nature humaine permet d'accéder aux sens et valeurs dont elle est porteuse pour les acteurs la créant, l'utilisant, voire la contestant. Ce faisant, la biographie d'innovation se veut aussi une voie pour accéder aux valeurs incorporées et nous connaître nous-même. En accédant à la question du sens de l'innovation, la biographie d'innovation apparaît un moyen de s'affranchir du registre de l'aliénation supposée causée par une incompréhension du mode d'existence des objets techniques [SIM 58].

Ces trois enseignements constitutifs de l'approche biographique concourent selon nous à l'élaboration d'une culture technique accessible aux concepteurs, usagers ou citoyens (Anonyme, à paraître). Cette culture technique a une portée agissante [DEF 93] et permet une forme de réflexivité sur les artefacts qu'en tant qu'Humains nous créons, nous utilisons, etc. Dans le cas présent, la biographie du TEL permet à un concepteur de saisir l'importance des filiations, des rapprochements avec d'autres dispositifs techniques et/ou d'autres procédés de filage, et de comprendre ainsi l'importance de la rationalité créative dans l'exercice de sa profession. Cela lui permet aussi de comprendre l'importance du terreau économique et social dans lequel il navigue et d'éviter de n'envisager l'innovation seulement commet un résultat issu du laboratoire de R\&D hors-sol.

\section{Bibliographie et sources}

Cet article repose sur une exploitation des documents trouvés dans les archives de Saint Gobain. Ces documents variés sont cités ci-dessous :

\section{Sources orales}

Interview Jean Battigelli, 25 mai 2018.

\section{Sources écrites (émanant des Archives de Saint-Gobain ou communiquées par la direction générale)}

[BAI 59] Bailly C., «La fibre de verre», Revue Isolation et revêtements, $\mathrm{n}^{\circ} 20$, septembre/octobre, Editions Techniques Riegel, [DOC ISO 49.03 (4003)], 1959.

[BAT 06] Battigelli J., Histoire du TEL, Première partie (jusqu'en 1960) : La genèse du TEL, document interne, [DOC ISO 00056.01], 2006.

[BAT 88] Battigelli J., Evolution du procédé TEL, document interne, [DOC ISO 00056.07], 1988.

[BER 05] Berthon L. Interview à l'occasion des 50 ans du TEL, document interne, [DOC ISO 00060.11], 2005.

[BUL 67] Bulletin d'information., « Notes historiques sur l'isolation », Article bulletin d'information, 08 mai 1967, [CSG HIST 0083001], 1967.

[COM 57] Compagnie de Saint-Gobain - Usine de Rantigny, La fibre de verre, juin 1957, document interne, [Doc ISO 4903 (4003)], 1957.

[DAR 07] Darche Y., «La petite histoire de la laine de verre », Amicale des retraités de Saint-Gobain, document interne, 2007.

[DIR 57] Direction générale des glaceries., Compte-rendu des réunions des chefs de groupements et chefs des services centraux, 13-18 mai 1957, document interne, [CSG HIST 0007463], 1957.

[ETU 57] Etudes statistiques., Principes de fabrication de la laine de verre, 12 septembre 1957, document interne, [ISOVER 00023 07_1957/HIS 57 002], 1957. 
[EXP 57] Exposé du groupement VII aux réunions des chefs de groupements et chefs des services centraux, mai 1957, document interne [CSG HIST 00074 63], 1957.

[PEY 57] Peychès I., Notes pour servir à l'organisation d'une recherche, document interne, [ISO Rantigny, 00023/007], 1957.

[PEY 77] Peychès, I, Eléments pour contribuer à l'histoire de la fibre de verre à Saint-Gobain, document interne, $12 \mathrm{p}$. [DOC CSG 58 8], 1977.

[TOL 80] Tolédano M., Marcel Lévecque, notre ami, [DOC SG 114.01], 1980.

[VIL 05] Villain R., 50 ans du procédé TEL. Témoignage. Interview de Raymond Villain, chef de four et responsable de l'usine d'Orange, document interne, [DOC ISO $0006011 \mathrm{itw}$ ], 2005.

\section{Brevets}

[Brevet FR 512925], Procédé et appareil pour filer le verre, délivré le 26 octobre 1920, inventeur Gedeon von Pazsiczky.

[Brevet FR 9819650], Perfectionnement à la fabrication de fibres, notamment de fibres de verre, délivré le 24 janvier 1951, Saint Gobain.

[Brevet FR 983422], Procédé et appareil pour la fabrication des fibres, notamment de fibres de verre, délivré le 14 février 1951, Saint Gobain.

[Brevet FR 994865 A], Procédé et dispositifs pour la fabrication de fibres à partir de matières thermoplastiques telles que le verre, délivré le 14 aout 1951, Saint Gobain.

[Brevet US 1427014], Apparatus for the production of spun glass, du 22 août 1922, inventeur Gedeon von Pazsiczky.

[Brevet US 2206058], Manufacture of glass wool, délivré le 2 juin 1940, inventeurs Games Slayter. Joen. H. Thomas, Owens Corning.

[Brevet US 2624912 A], Process and apparatus for the production of fibers from thermo-plastics, délivré le 13 janvier 1953, inventeurs Pierre Heymes, Ivan Peyches, Saint Gobain.

\section{Bibliographie}

[BER 07] Berneron-Couvenhes M-F, « La concession des services maritimes postaux au xixe siècle. Le cas exemplaire des Messageries Maritimes », Revue économique, 1(58), p. 259-276, 2007.

[CAL 94] Callon M., «L'innovation technologique et ses mythes », Gérer et comprendre, 34, p. 4-17, 1994.

[CHO 60] Choffel J., Saint-Gobain, du miroir à l'atome, Plon, Paris, 1960.

[CHO 18] Chouteau M., Forest J., Nguyen C., «La créativité au cœur du procédé TELde Saint-Gobain? Analyse de quelques discours institutionnels à travers le temps (1957-2007) », Actes du Congrès de la SFSIC 2018, Volume 2, p. 496-511, 2018.

[CHO 17] Chouteau M., Forest J., Nguyen C., 2017, «Quand la culture d'innovation fait écran à la culture technique », Technologie et innovation, volume 17(4), ISTE OpenScience, mis en ligne le 5 septembre 2017, https://www.openscience.fr/Quand-la-culture-d-innovation-fait-ecran-a-la-culture-technique.

[DEF 93] Deforge, Y., De l'éducation technologique à la culture technique, ESF Editeur, Paris, 1993.

[FOR 17a] Forest J., Creative rationality and innovation, WILEY-ISTE Editions, London, 2017.

[FOR 17b] Forest J., « Il n’y a pas de génie solitaire », La Recherche, Chronique Innovation, décembre, № 530, 2017.

[FOR 03] Forest J., Micaelli J-P., Artificialisme, Presses Polytechniques Universitaires Romande, Belfort, 2003.

[ISO 07] ISOVER., Saint-Gobain et la laine de verre. Histoire d'une réussite internationale, Saint-Gobain/Textuel, 2007.

[LER 69] Le Roux P., «L'équipement des Français en biens durables, fin 1968 », Economie et statistiques, 3, p. 6568, 1969.

[SIM 58] Simondon G., Du mode d'existence des objets techniques, Aubier, Paris, 1958. 